\title{
LOW-FREQUENCY MICROINSTABILITIES IN THE PLT TOKAMAK
}

E. MAZZUCATO

\section{PLASMA PHYSICS LABORATORY}

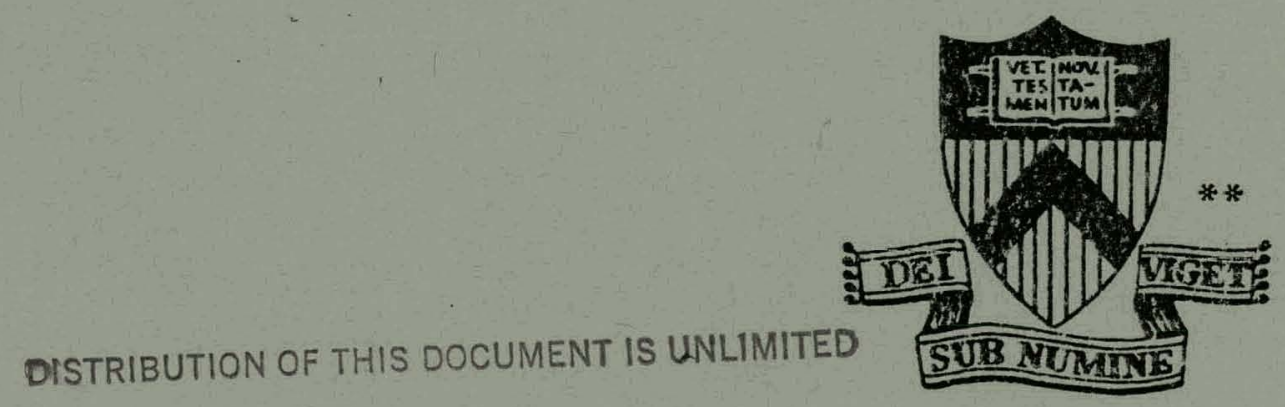

\section{PRINCETON \\ UNIVERSITY PRINCETON, NEW JERSEY}

This work was supported by U. S. Energy Research and Develocment Administration Contrast EY-76-C-02-3073. Reproduction, translation, publication, use and disposaz, in whole or in part, by or for the United States Governanent is permitted. 


\section{DISCLAIMER}

This report was prepared as an account of work sponsored by an agency of the United States Government. Neither the United States Government nor any agency Thereof, nor any of their employees, makes any warranty, express or implied, or assumes any legal liability or responsibility for the accuracy, completeness, or usefulness of any information, apparatus, product, or process disclosed, or represents that its use would not infringe privately owned rights. Reference herein to any specific commercial product, process, or service by trade name, trademark, manufacturer, or otherwise does not necessarily constitute or imply its endorsement, recommendation, or favoring by the United States Government or any agency thereof. The views and opinions of authors expressed herein do not necessarily state or reflect those of the United States Government or any agency thereof. 


\section{DISCLAIMER}

Portions of this document may be illegible in electronic image products. Images are produced from the best available original document. 
NOTICE

This report was prepared as an account of work sponsored by the United States Government. Neither the United States nor the United States Energy Research and Development Administration, nor any of their employees, nor any of their contractors, subcontractors, or their employees, makes any warranty, express

or implied, or assumes any legal liability or responsibility for the accuracy, completeness or usefulness of any information, apparatus, product or process disclosed, or represents that its use would not infringe privately owned rights.

Printed in the United States of America.

Available from

National Technical Information Service

U. S. Department of Commerce 5285 Port Royal Road

Springfield, Virginia 22151

Price: Printed Copy \$_*; Microficho $\$ 3.00$

*Pages

$1-50$

$51-150$

$151-325$

$326-500$

$501-1000$
NTIS

Selling Price

$\$ 4.00$

5.45

7.60

10.60

13.60 
Low-Frequency Microinstabilities in the PLT Tokamak

\author{
E. Mazzucato \\ Plasma Physics Laboratory, Princeton University \\ Princeton, New Jersey 08540
}

PPPL-1373

September 1977

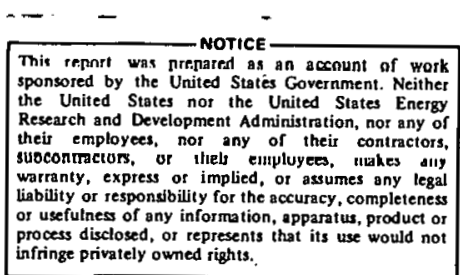

infringe privately owned rightss.

$\rightarrow \cdots$ 
Low-Frequency Microinstabilities in the PLT Tokamak

\author{
E. Mazzucato \\ Plasma Physics Laboratory, Princeton University \\ Princeton, New Jersey 08540
}

\begin{abstract}
A turbulence with wavelengths in the range $2-0.2 \mathrm{~cm}$ and with a spectrum in the range of frequencies of drift waves has been observed in the PLT discharge with scattering of microwaves. The total density fluctuation was $\tilde{n}_{e} / \bar{n}_{e} \simeq 5 \times 10^{-3}-10^{-2}$. We estimate that this turbulence can cause an anomalous transport much larger. than that predicted by the neoclassical theory.

As the PLT discharge developed a central minimum of the electron temperature, an increase of the turbulence level was observed with a very strong poloidal localization on the outside of the plasma torus.
\end{abstract}


I. INTRODUCTION

In spite of vast amounts of work, the confinement of electrons in low- $\beta$ toroidal magnetic configurations is still an unresolved problem. The reason is not only our limited knowledge of this phenomenon but also the difficulty of interpreting the experimental data in a unique way. This is particularly true in the field of tokamaks where a single plasma parameter, the toroidal electric current, not only determines the equilibrium and the stability of the magnetic configuration but also provides the only effective way of heating the electrons.

To explain the behaviour of electrons in present tokamak experiments we must invoke anomalous losses. ${ }^{1,2}$ A variety of microinstabilities can be excited in a MHD-stable tokamak and cause an increase of the plasma transport. The most important class are the drift-type modes which are driven by the plasma free energy associated with the density and temperature gradients. ${ }^{3,4}$

Drift waves can be classified into three major groups according to the plasma collisionality regime. At the low temperature end of the tokamak parameter range we have the collisional or dissipative drift modes ${ }^{3}$ which are destabilized by coulomb collisions $\left(\nu_{e}\right)$. The existence of this mode requires that $\nu_{e}>k_{\|} v_{e}$. where $k_{\|}$is the wave vector component along the magnetic field and $v_{e}$ is the electron thermal velocity. These modes can give rise to a Bohm-like diffusion rate but fortunately they can exist only at the edge of present-day tokamaks.

At lower collision frequencies, where $\nu_{e}<k_{\|} v_{e}$, we have the collisionless drift modes. 5 In this regime the plasma free energy 
is available to drift waves through the inverse electron Landau damping. These modes are destabilized by finite ion gyroradius effects and by density and temperature gradients in opposite di-

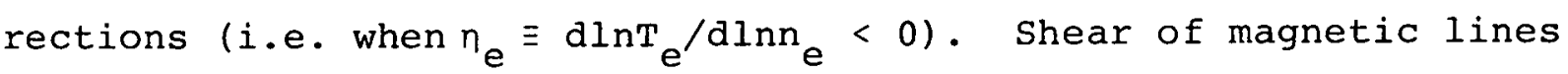
has a stabilizing effect. The original estimates ${ }^{6}$ indicated that these modes are stabilized when $r_{n} / L_{s}>\left(m_{e} / m_{i}\right)^{1 / 3}$ [where $r_{n}$ $=\left(d \operatorname{lnn} e^{/ d r}\right)^{-1}$ is the density scale length, $L_{s}=\left[\left(\varepsilon / q^{2}\right)(d q / d r)\right]^{-1}$ is the shear length, $\varepsilon=r / R$ is the aspect ratio and $q$ is the $M H D$ safety factor]. Such levels of shear are not available in tokamaks. Recently Taylor ${ }^{7}$ and Cordey and Hastie ${ }^{8}$ have shown that the effect of shear in tokamaks is weakened by the variation of the magnetic field properties around the magnetic surface which tends to localize drift modes in both poloidal and radial directions.

With rising temperatures the plasma enters into a regime where some particles can experience the $1 / R$ dependence of the toroidal magnetic field and they become trapped into the local magnetic mirrors. The presence of these trapped particles makes the free plasma energy more accessible to drift waves and a new group of modes becomes unstable. Among these instabilities the one which is of interest for present tokamak experiments is the dissipative trapped-electron mode ${ }^{9}$ which is ariven by positive temperature gradient (i.e. $\eta_{e}>0$ ) together with collisional dissipation. These instabilities can give rise to a plasma transport which is much larger than that produced by classical coulomb collisions. Their effect can be visualized as a random walk of particles across the magnetic surfaces under the influence of collisions and $\overline{\mathrm{E}} \times \overline{\mathrm{B}}$ drifts. The exact estimate of this effect needs a 
self-consistent calculation of the amplitude of these instabilities which presents formidable difficulties. We must therefore rely on simplified models of this phenomenon. Simple stochastic arguments indicate that the diffusion coefficient $D$ can be expressed as $D \simeq(\Delta r)^{2} / \Delta t$, in terms of an effective step size $\Delta r$ and the associated time interval $\Delta t$. Approximating the correlation time $\Delta t$ with the growth time $\gamma^{-1}$ of the instability and the step size $\Delta r$ with the average radial wavelength $\mathrm{k}_{r}^{-1}$, we get the familiar result ${ }^{3}$

$$
i=\gamma / k_{r}^{2} .
$$

Another approach is the quasilinear procedure of calculating the particle radial flux $\Gamma$ by averaging the drift equations of motion over velocities and the fast time scale of the fluctuations. This gives

$$
\Gamma \simeq-\sum_{\mathrm{k}} \tilde{\mathrm{n}}_{\mathrm{k}} \nabla \tilde{\phi}_{\mathrm{k}} \times \underline{\mathrm{BC}}^{\mathrm{B}} \mathrm{B}^{2}
$$

where $\tilde{n}_{k}$ and $\tilde{\phi}_{k}$ are the amplitudes of the density and the electrostatic potential fluctuations which are induced by the $\mathrm{k}$-mode. Since for drift waves $\omega_{k}\left(\tilde{n}_{k} / n_{e}\right) \simeq \omega_{*}\left(e \tilde{\phi} / T_{e}\right) \quad$ [with $\omega_{k} \simeq \omega_{*}+i \gamma_{k}$. $\left.\omega_{*}=\left(k_{\theta} \mathrm{CT}\right)\right) /\left(e B r_{n}\right)$ and $k_{\theta}$ the poloidal wave vector $]$ we can express the anomalous flux in terms of a diffusion coefficient given by

$$
D \simeq \sum_{k}\left(e \tilde{\phi}_{k} / T_{e}\right)^{2} r_{n}^{2} \gamma_{k}
$$

This expression coincides with that of Eq. (1) if we assume that $\left(e \tilde{\phi}_{k} / T_{e}\right) \simeq 1 /\left(k_{r} r_{n}\right)$. The physical meaning of this relation is that the unstable mode saturates when the gradient of the density 
fluctuation becomes equal to the equilibrium density gradient. ' Liu et al. ${ }^{10}$ arrived at the same result by equating the available free plasma energy to the drift wave energy density.

Drift waves contribute not only to the particle transport but also to the thermal transport both, by convection and by conduction. The convective part can be readily obtained multiplying Eq. (2) by the factor $1.5 \mathrm{~T}$. The conduction part is obtained from the $\mathrm{v}^{2}$-moment of the fluctuating distribution function. The relative values of conduction and convection depend on the particular mode we consider but in general for normal profiles they have the same order of magnitude.

In the regime of operation of present tokamak experiments we expect the anomalous transport to be mainly affected by collisionless drift waves for which $\gamma \propto \omega_{*}^{2} /\left(k_{\|} v_{e}\right)$. By equating the ohmic input to the largest anomalous losses, which are given by Eq. (1) with $k_{\theta}^{-2}=k_{r}^{-2}=\rho_{i e}^{2} \equiv m_{i} T_{e}(c / e B)^{2}$, we obtain $T_{e} \propto n_{e}^{-1 / 4}$. This result is in agreement with the weak dependence of the electron temperature from the plasma density which is found experimentally. 2

For a plasma with the characteristics of the PLT discharge ${ }^{11}$ $\left(B=30 \mathrm{kG}, r_{n} \simeq 20 \mathrm{~cm}, T_{e} \simeq 1 \mathrm{keV}\right)$ these estimates give $\left(\mathrm{e} \tilde{\phi} / \mathrm{T}_{\mathrm{e}}\right)$ $\simeq(\tilde{n} / n) \simeq 5 \times 10^{-3}$ (with $k_{r} \simeq 10 \mathrm{~cm}^{-1}$ ) and a particle confinement time $\tau_{p} \simeq 300 \mathrm{msec}$ (with $\gamma / \omega_{*} \simeq 0.2$ ). For the electron energy confinement time we get $\tau_{E} \simeq 100 \mathrm{msec}$, to be compared to the value of $40 \mathrm{msec}$ which was experimentally obtained for the gross electron energy confinement time. 11

These'simple arguments indicate how a small level of electrostatic turbulence can affect the plasma confinement. Recent experiments on the $\operatorname{ATC}^{12,13}$ and $\operatorname{TFR}^{14}$ tokamaks have proved the 
existence of such turbulence. In this paper we present some additional results which were obtained in the PLT tokamak.

\section{EXPERIMENTAL APPARATUS}

Scattering of microwaves was used for studying low frequency microinstabilities in PLT. A block diagram of the experimental arrangement is shown in Fig. 1 .

An array of identical antennae (gain $\simeq 27 \mathrm{db}$ ) was used for launching the output of an Extended Interaction oscillator and for collecting the waves which were scattered by fluctuations of the plasma density. The incident wave had a frequency $\nu_{0}=140 \mathrm{GHz}$, a power $\mathrm{P}_{0}=5 \mathrm{~W}$ and it was polarized with the electric field parallel to the toroidal magnetic field. Both the launching and the receiving antennae were located on the same poloidal plane and the scattering angle varied from $0^{\circ}$ to $60^{\circ}$.

A homodyne detection system was used in which the received wave was mixed with a larger reference wave from the microwave oscillator and it was detected by a Low-Barrier Schottky Diode. The resultant $r$.f. signal was amplified by an amplifier with a bandwidth of $\mathrm{I} \mathrm{MHz}$ and it was Fourier analyzed by a commercial spectrum analyzer with a frequency resolution of $10 \mathrm{kHz}$.

The process of incoherent scattering of electromagnetic waves by plasma density fluctuations can be characterized by the differential cross section ${ }^{15}$

$$
u=r_{0}^{2} s(\underline{k}, \omega)
$$

where $r_{0}=e^{2} / \mathrm{mc}^{2}$ is the classical electron radius and $\mathrm{s}(\underline{\mathrm{k}}, \omega)$ is 
the spectral density of electron density fluctuations which is given by

$$
S(\underline{k}, \omega)=\lim _{\substack{V \rightarrow \infty \\ T \rightarrow \infty}} \frac{2}{V T}\left|n_{e}(\underline{k}, \omega)\right|^{2},
$$

with

$$
n_{e}(\underline{k}, \omega)=\int_{T} d t \int_{V} d \underline{r} \exp [-i(\omega t+\underline{k} \cdot \underline{r})] n_{e}(\underline{r}, t) \text {. }
$$

The frequency $\omega$ and the wave vector $\underline{k}$ must satisfy the energy and momentum conservation, that is $\omega=\omega_{s}-\omega_{i}$ and $\underline{k}=\underline{k}_{s}-\underline{k}_{i}$, where the subscripts $s$ and $i$ refer to the scattered and incident wave respectively.

The total mean-square density fluctuation is given by

$$
\left\langle\left.\tilde{\mathrm{n}}_{\mathrm{e}}\right|^{2}\right\rangle=(2 \pi)^{-4} \int \mathrm{S}(\underline{k}, \omega) \mathrm{dk} \mathrm{d} \omega
$$

where the region of integration is the entire $(\underline{k}, \omega)$ space.

The power $\mathrm{dP}_{\mathrm{s}}$ which is scattered into the receiving antenna per unit frequency by the plasma volume $d v$ is

$$
\mathrm{dP}_{\mathrm{s}}=\sigma \mathrm{P}_{\mathrm{i}} \Omega_{\mathrm{s}} \mathrm{dV}
$$

where $P_{i}(\underline{r})$ is the incident power density and $\Omega_{s}(\underline{r})$ is the effective solid angle of reception. We can express these quantities in terms of the antenna radiation patterns $F(\alpha, \beta)$ [where $\alpha$ and $\beta$ are two angular coordinates which specify the direction of the wave and $F(0,0)=1]$. Using subscripts $t$ and $r$ for the transmitter 
and the receiver respectively, we have $P_{i}(\underline{r})=P_{0} G F\left(\alpha_{t}, \beta_{t}\right) / 4 \pi\left|\underline{r}_{t}-\underline{r}\right|^{2}$ and $\Omega_{S}(\underline{r})=\lambda_{0}^{2} G F\left(\alpha_{r}, \beta_{r}\right) / 4 \pi\left|\underline{r}_{r}-\underline{r}\right|^{2}$, where $G=4 \pi / \int F(\alpha, \beta) d \Omega$ is the maximum gain of the antenna and $\lambda_{0}=c / \nu_{0}$.

Integrating Eq. (6) over the plasma volume we obtain

$$
\frac{P_{s}}{P_{0}}=r_{0}^{2}\left\langle S(\underline{k}, \omega)>\left(\frac{G \lambda_{o}}{4 \pi}\right)^{2} \int \frac{F\left(\alpha_{t}, B_{t}\right) F\left(\alpha_{r}, B_{r}\right)}{\left|\underline{r}_{t}-r\right|^{2}\left|\underline{r}_{r}-\underline{r}\right|^{2}} d V .\right.
$$

The function $\langle S(\underline{k}, \omega)\rangle$ which appears in Eq. (7) is an average value of $S(\underline{k}, \omega)$ over the scattering volume and a range of $\underline{k}$-vectors which is specified by the given beam geometry. In this experiment the $\mathrm{k}$-resolution was $\Delta \mathrm{k} \simeq \pm 2 \mathrm{~cm}^{-1}$.

All but two of the scattering geometries we used had their centerpoint [defined by the equation $F\left(\alpha_{t}, \beta_{t}\right) F\left(\alpha_{r}, B_{r}\right)=1$ ] at $r=22 \mathrm{~cm}$ and $\theta=90^{\circ}$. Here the coordinates $(r, \theta)$ are the usual minor radius and poloidal angle respectively $(\theta=0$ being the outward direction). The average $\underline{k}$-vectors in these geometries were mainly along the poloidal direction. The other two geometries we used had the centerpoint at $\mathrm{r}=14 \mathrm{~cm}, \theta=-54^{\circ}$ and $\mathrm{r}=15 \mathrm{~cm}$, $\theta=-135^{\circ}$ respectively. They were chosen as symmetrical as possible with respect to the plane which was perpendicular to the line $\theta=0^{\circ}$ and contained the center of the plasma cross section. The part of their scattering regions where $F \geqslant 0.5$ for both the transmitter and the receiver are shown in Fig. 2 together with the equivalent region of one of the other geometries used. In all the three cases $k \simeq 10 \mathrm{~cm}^{-1}$. For larger values of $k$ the spatial resolution was better than that shown. in Fig. 2 while the opposite was true for smaller values of $k$. 
Equation (7) is valid only in free-space. Refraction of waves by the average gradients of the plasma density modifies the functions $\mathrm{P}_{i}(\underline{r})$ and $\Omega_{\mathrm{s}}(\underline{r})$ of $\mathrm{Eq}$. (6). We have calculated this effect for the case of parabolic plasma density profiles and we have found that for central electron densities smaller than $10^{14} \mathrm{~cm}^{-3}$ refraction modifies the value of $P_{S}$ by less than $15 \%$. In this range of densities the main effect of refraction is that of changing the position and the shape of the scattering volume with the result that the contribution of the plasma edge becomes more important as the plasma density increases. Since all our measurements were performed in discharges with $\mathrm{n}_{\mathrm{e}} \ll 1 \times 10^{14} \mathrm{~cm}^{-3}$, we have neglected the effect of refraction on $P_{S}$ while we have taken into account that on the $\underline{k}$-vectors.

\section{EXPERIMENTAL RESULTS}

The measurements described in thị paper were performed in deuterium discharges with a minor radius $r=40 \mathrm{~cm}$, a major radius $\mathrm{R}=132 \mathrm{~cm}$ and a toroidal magnetic field $\mathrm{B}_{\mathrm{t}}=32 \mathrm{kG}$. During their quasi-stationary phase (typically for it $>200$ msec) the toroidal electric current was $400 \mathrm{kA}$ and the electron density had a central value of $4 \times 10^{13} \mathrm{~cm}^{-3}$ with a radial distribution not far from a parabolic profile. In thịs range of parameters two different types of discharges were produced in the PLT tokamak. Their typical electron temperature profiles, which were measured with 'Thomson scattering of laser light at $t=300 \mathrm{msec}$, are shown in F.ig. 3. One type of discharge exhibited the standard peaked temperature profile with central values of $\sim 1500 \mathrm{eV}$. On the con:trary, the other type developed a central depression with maxima 
of $600-800 \mathrm{eV}$ at $\mathrm{r}=15-20 \mathrm{~cm}$ and central minima of $200-300 \mathrm{eV}$. The two profiles were very similar in the outer region $r>20 \mathrm{~cm}$.

The time evolution of the temperature of a "hollow" discharge is shown in Fig: 4. After the early turbulent stage that follows the formation of the discharge; the electron temperature profile. assumes a peaked shape that lasts for about $100 \mathrm{msec}$. At this point the central temperature begins to collapse. Once formed the hollow temperature profile shows a remarkable stability up to the end of the discharge pulse. The causes of this phenomenon are still under investigation. A necessary but not sufficient condition seems to be the presence of heavy impurities in the central region of the plasma column. 16

Discharges with peaked $T_{c}$ profiles had peaked $T_{i}$ profiles too, with central values $T_{i 0} \simeq 600-900 \mathrm{eV}$; those with hollow $T_{e}$ radial distributions exhibited a small central depression of $T_{i}$ with central values $\mathrm{T}_{\text {io }} \simeq(0.8-0.9) \mathrm{T}_{\dot{\mathrm{i}}}^{\max } \cong 500 \mathrm{eV}$.

The values of $z_{\text {eff }}(=5-8)$ as well as those of the gross electron energy confinement time ${ }_{E}(\simeq 10 \mathrm{msec})$ were very similar in the two types of discharges..

The frequency spectrum of the scattering signal produced by density fluctuations with $\mathrm{k} \simeq 10 \mathrm{~cm}^{-1}$ is shown in Fig. 5. For comparison the spectrum produced by similar fluctuations in the ATC tokamak ${ }^{12}$ is also shown. The amplitudes of these signals are proportional to $[S(\underline{k}, \omega)]^{1 / 2}$ since the crystal detectors were operating in their square-law region. These measurements were made in a discharge with the peaked $\mathrm{T}_{\mathrm{e}}$-profile of Fig. 3 and the central points of the scattering regions were located at $r=22 \mathrm{~cm}$, $\theta=90^{\circ}$ (see Fig. 2). The frequency width at half intensity of 
the spectral density $\mathbf{s}(\underline{k}, \omega)$ is plotted as a function of the wavenumber $\mathrm{k}$ in Fig. 6 (o-points) together with similar results from the ATC tokamak ${ }^{13}$ (o-points).

The spectral density in the $k$-space, $S(\underline{k})=(1 / 2 \pi) \int_{-\infty}^{+\infty} S(\underline{k}, \omega) d \omega$, is plotted in Fig. 7. as a function of the wave number $\mathrm{k}$ for the discharge with the peaked $\mathrm{T}$-profile of Fig. 3. The o-points are for scattering volumes with centerpoints at $\theta=90^{\circ}$; the $*$-points are for the other two scattering geometries (see rig. 2). The fluctuations in the outside scattering region $\left(\theta=-54^{\circ}\right)$ were larger than those in the inside region $\left(\theta=-135^{\circ}\right)$. The e-points are the results obtained in a similar ATC discharge. 13

The values of $\mathrm{S}(\underline{\mathrm{k}})$ for the casse of hollow $\mathrm{T}_{\mathrm{e}}$-profile are plotted in Fig. 8. In this type of discharge we measured a larger level of turbulence. This is clearly shown in Fig. 9 where the frequency spectra of the signals received from the outside scattering region are shown for the two types of discharges. The amplitude in the spectral region around $(\omega / 2 \pi)=100 \mathrm{kHz}$ is much larger in the case of hollow $\mathrm{T}_{\mathrm{e}}$-profile. The corresponding values of $\mathrm{S}(\underline{\mathrm{k}})$ in the two types of discharges are plotted in Fig. 10 as a function of time. These results show very clearly how the departure of the two values of $\mathbf{S}(\underline{k})$ starts at the moment the central value of $\mathrm{T}$ e begins to collapse.

The ratio of the outside value of $\mathrm{s}(\underline{\mathrm{k}}, \omega)$ to the inside one for $(\omega / 2 \pi)=100 \mathrm{kHz}$ is plotted as a function of time in Fig. Il for the two types of discharges. These data indicate that the eribancement of the turbulence caused by the hollow $\mathrm{T}_{\mathrm{e}}$-distribution has a poloidal structure with a much stronger localization on the outside cif the plasma torus. 


\section{DISCUSSION OF THE EXPERIMENTAL . RESULTS}

The turbulence observed in the PLT device, as well as that previously observed in the ATC tokamak, 12,13 is in the range of frequencies of drift waves. As a matter of fact the largest frequencies which were observed were 2-3 times larger than those predicted by the linear theory of drift waves. Nevertheless one may argue that, if what we observed is the saturated level of a turbulence which was produced by drift-waves, there is no reason for expecting just the frequencies predicted by the linear theory. Moreover, a poloidal plasma rotation, as that observed in the ST device, 17 can be a further cause of discrepancy between the measured frequency and that of the plasma fluctuation.

To settle the question if the observed turbulence was produced by arift waves, it is of great interest to compare the range of frequencies of the turbulence that was observed in $\mathrm{ATC}^{12,13}$ and PL'I. Although these two devices have different characteristics, their plasmas have very similar parameters. We have $B_{t}^{\mathrm{PLT}} / \mathrm{B}_{t}^{\mathrm{ATC}}$ $\simeq 2.0, \mathrm{r}_{\mathrm{n}}^{\mathrm{PLT}} / \mathrm{r}_{\mathrm{n}}^{\mathrm{ATC}} \simeq \mathrm{r}^{\mathrm{PLT}} / \mathrm{r}^{\mathrm{ATC}}=2.3, \mathrm{~T}_{\mathrm{e}}^{\mathrm{PLT}} / \mathrm{T}_{\mathrm{e}}^{\mathrm{ATC}} \simeq 1.5$ and therefore $\omega_{*}^{A T C} / \omega_{*}^{\mathrm{PLT}} \simeq 3$ which is just the ratio of the frequencies detected in these two devices as it is shown in Figs. 5 and 6.

If we assume that $k_{r} \simeq k_{\theta}<k_{\|}$by integrating the function $\mathrm{S}(\mathrm{k})$ we: obtain $\tilde{\mathrm{n}}_{e} / \overline{\mathrm{n}}_{e} \simeq 5 \times 10^{-3}-10^{-2}$. This value is in good agreement with the quasi-linear estimate $\tilde{n}_{e} / \bar{n}_{e} \simeq 1 / k_{r} r_{n}$.

The shape of $S(\underline{k})$ in PLT is different from that previously found in ATC. While $\mathrm{S}(\underline{\mathrm{k}})$ showed in ATC a maximum in the region where $\mathrm{k}_{\theta} \rho_{i} \simeq 0.5$, the $\mathrm{S}(\underline{\mathrm{k}})$ measured in PLT decreased monotonically with increasing values of $\mathrm{k}$ and it extended to larger values of $\mathrm{k}$. 
However, one must point out that since $\mathrm{T}_{i}^{\mathrm{PLT}} / \mathrm{T}_{i}^{\mathrm{ATC}} \simeq 3-4$ and $\lambda_{0}^{\mathrm{ATC}} / \lambda_{\mathrm{O}}^{\mathrm{PLT}}=2$, fluctuations with $\mathrm{k} \simeq\left(2 \rho_{i}\right)^{-1}$ were detected in PLT only at small scattering angles and therefore in conditions of very large scattering regions where the contribution of the exterior part of the plasma column to the scattered power was very important.. The different shape of $\mathrm{S}(\underline{k})$ at small values of $\mathrm{k}$ could just be due to the different spatial averaging which was used in the two experiments.

From the quasi-linear estimate of the anomalous transport produced by 'the observed turbulence [Eq. (3)] we get a value of 50-100 msiec for the electron confinement time of the PLT discharge. Since we measured a gross electron energy confinement time of 10 msec, we conclude that the observed turbulence was not the dominant cause of energy 1oss. Nevertheless the estimated anomalous transport is much larger than that predicted by the neoclassical theory.

The two types of discharges we studied had identical characteristics in the region $r>20 \mathrm{~cm}$. Therefore the increase of the level of turbulence we detected in the second type of discharge had to be generated in the central region of the plasma column where the radial distribution of $\mathrm{T}_{\mathrm{e}}$ had a large depression which caused $n_{e} \equiv a \ln T_{e} / d l n n_{e}<0$. A negative value of $n_{e}$ is a cause of instability for collisionless drift modes ${ }^{18}$ (i.e. universal mode) while it has a stabilizing effect on the dissipative trappedelectron mode. 9 The increase of the turbulence level in the hollow discharge must be considered clear evidence that the observed fluctuations were not caused by trapped-particle effects.

The values of $\mathrm{S}(\underline{\mathrm{k}})$ which are plotted in Fig. 8 were averaged over larger scattering volumes which contained both plasma regions with $n_{e}=0$ and $n_{e}>0$. Therefore the local values of $S(\underline{k})$ in the 
region with $\eta_{e}<0$ were considerably larger than those shown in Fig. 8 .

This turbulence caused an anomalous transport towards the plasma center. This was confirmed by bolometric measurements which showed that the amount of energy radiated by impurities in the central region of the plasma column was much larger than the ohmic input. 19 It is not inconceivable that this turbulence might have caused an anomalous resistivity as well. 20

\section{v. CONCLUSION}

A turbulence with a spectrum in the range of frequencies of drift waves has been observed in the PLT discharge. The total fluctuating amplitude, $\tilde{n}_{\theta} / \bar{n}_{\theta} \simeq 5 \times 10^{-3}-10^{-2}$, is in agreement with the value predicted by the quasi-linear theory but it is not suf-. ficient to explain the extremely large losses of this discharge that were mainly due to a large amount of impurities. Nevertheless we estimate that the observed level of turbulence can cause an anomalous transport which is much larger than that predicted by the neoclassical theory and which is capable of carrying the plasma energy to the boundary in about $100 \mathrm{msec}$. 


\section{ACKNOWLEDGMENTS}

The author wishes, to thank N. Bretz, D. Dimock and D. Johnson for providing the results of the Thomson scattering measurements. This work was supported by: the U. S. Energy Research and Development Administration, contract EY-76-C-02-3073. 


\section{REFEREṄCES}

${ }^{1}$ L. A. Artsimovich, Nucl. Fusion 12,215 (1972):

${ }^{2} \mathrm{H}$ : P. Furth, Nucl. Fúsion 15, 67 (1975).

${ }^{3}$ B. B. Kadomtsev and O. P. Pogutse, in Review of Plasma Physics, edited by M. A. Leontovich (Consultant Bureau, New York, 1970), Vol. 5, , p. 249 .

${ }^{4} \mathrm{~B}$. Coppi and G. Rewoldt, in Advances in Plasma Physics, edited by A. Simon and W. Thompson (Wiley, New York, 1976), Vol. 6, p. 421 .

${ }^{5}$ N. A. Krall and M. N. Rosenbluth, Phys. Fluids $\underline{8}, 1488$ (1965).

${ }^{6}$ L. D. Pearlstein and H. L. Berk, Phys. Rev. Lett. 23,220 (1969) ..

7J. B. Taylor, in plàsma physics and Controlled Nuclear FuSion Research (International Atomic Energy Agency; Vienna, 1977), Vol. 2, p. 323.

${ }^{8} \mathrm{~J}$. G. Cordey and R. J. Hastie, Nucl. Fusion 17, 523 (1977).

${ }^{9}$ B. B. Kadomtsev and O. P. Pogutse, Sov. Phys. Doklady 14, 470 (1969).

${ }^{10}$ C. S. Liu, M. N. Rosenbluth, and C. W. Horton, Physs. Rev. Lett. 29,1489 (1972).

${ }^{11}$ D. Grove et al., in Plasma Physics and Controlled Nuclear Fusion Research (International Atomic Energy Agency, Vienna, 1.977). Vol. 1, p. 21.

12 E. Mazzucato, Phys. Rev. Lett. 36,792 (1976).

${ }^{13}$ R. J. Goldston, E. Mazzucato, R. E. Slusher and C. M. Surko, in Plasma Physics and Controlled Nuclear Fusion Research (International Atomic Energy Agency, Vienna, 1977), Vol. 1, p. 371. 
${ }^{14}$ T.F.R. Group, in Plasma Physics and Controlled Nuclear Fusion Research (Ințernational Atomic Energergy Agency, Vienna, 1977), Vol. 1, p. 35.

${ }^{15}$ M. N. Rosenbluth and N. Rostoker, Phys. Fluids $\underline{5}, 776$ (1962). ${ }^{16} \mathrm{E}$, Hinnov and Mattioli (private communication). 17 J. C. Hosea, F. C. Jobes, R. L. Hickok, and A. N. Dellis, Phys. Rev. Lett. 30,839 (1973). ${ }^{18}$ A. A. Galeev, V. N. oraevskịi, and R. Z. Sagdeev, Sov. Phys. JETP $17,615 \quad(1963)$.

${ }^{19} \mathrm{H}$, Hsuan and $\mathrm{J}$, Schivell (prịvate communication). 20 B. Coppi and E. Mazzucato, Physs, Flụids 14, 134 (1971). 


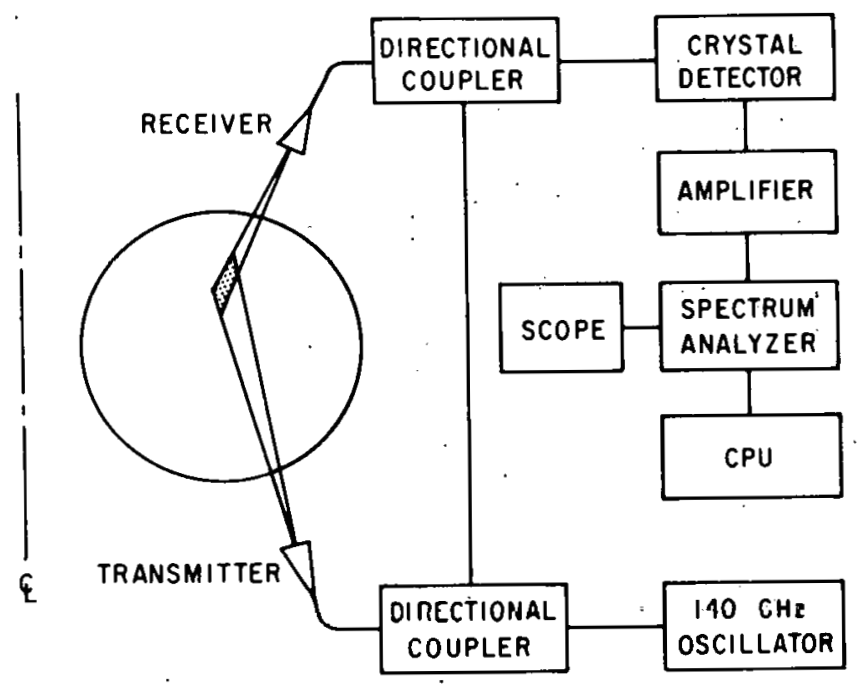

773651

Fig. 1. Block diagram of the experimental arrangement.

773652

Fig. 2. Some of the scattering regions that were used for detecting fluctuations with $k \simeq 10$ $\mathrm{cm}^{-1}$.

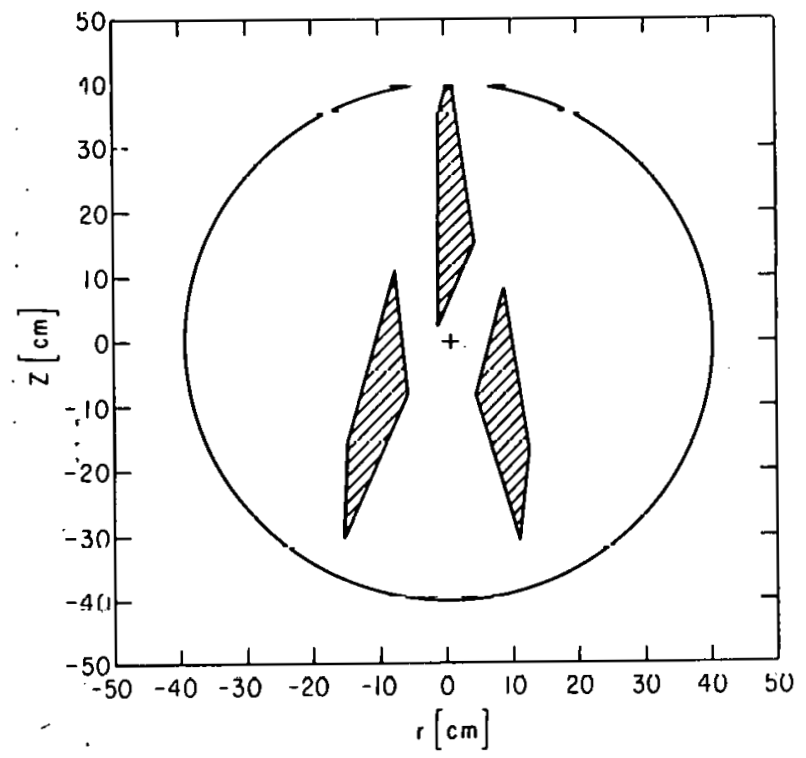


773655

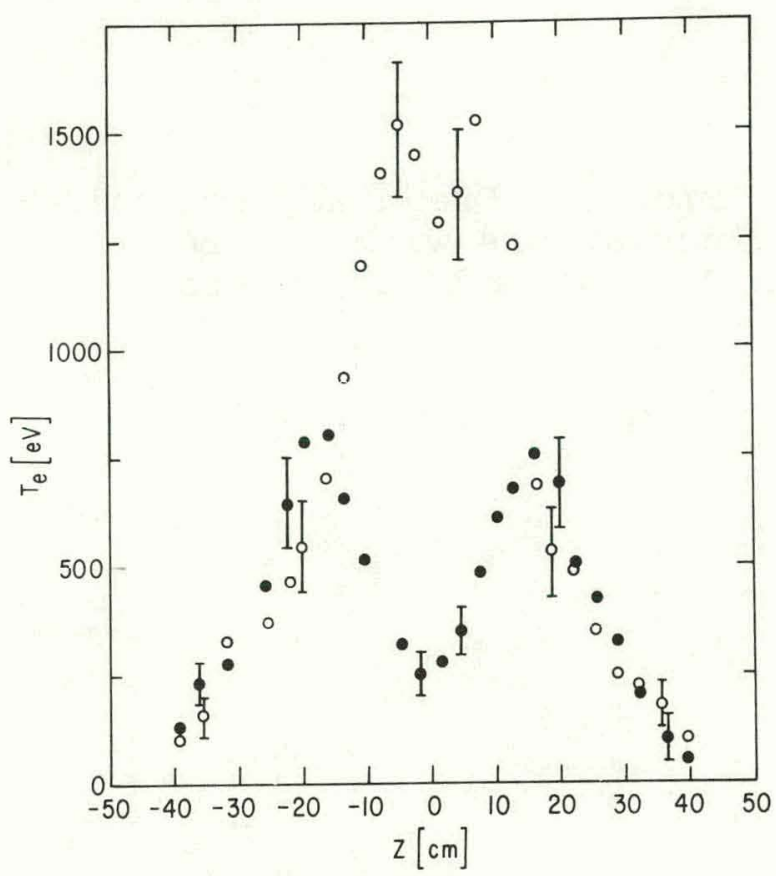

Fig. 3. $\mathrm{T}_{\mathrm{e}}$-profiles of two types of PLT discharges $(t=300 \mathrm{msec})$.

773704

Fig. 4. Time evolution of the hollow $\mathrm{T}_{\mathrm{e}}$-profile. The parameter of the curves is the time in msec; the discharge was formed at $t$ $=20 \mathrm{msec}$.

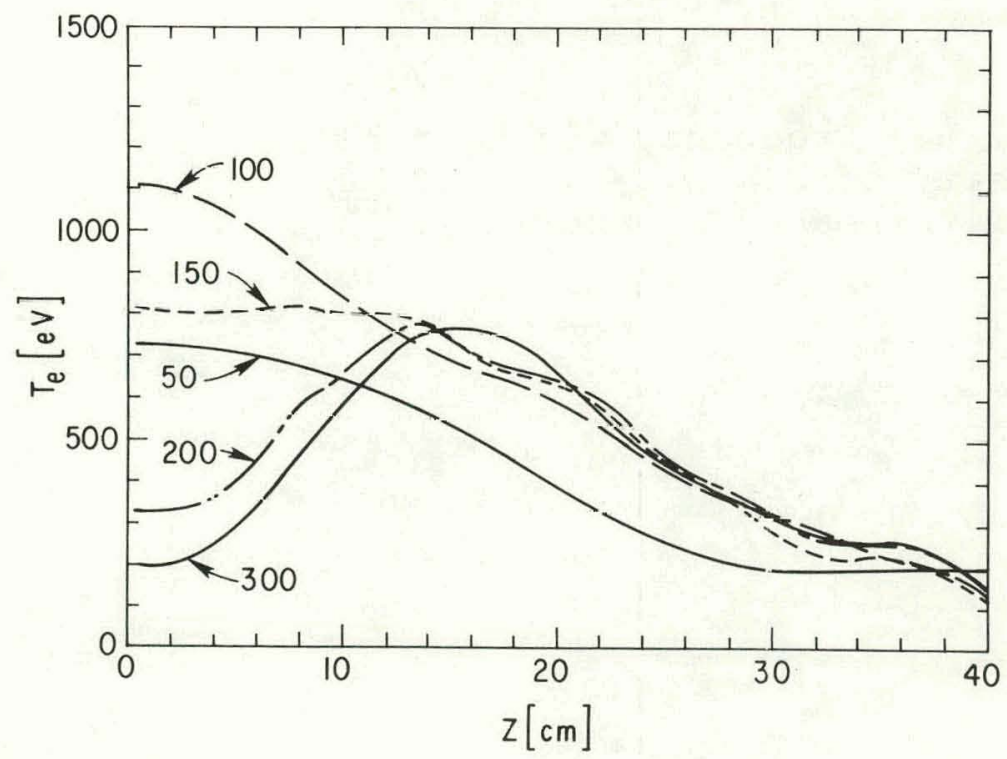



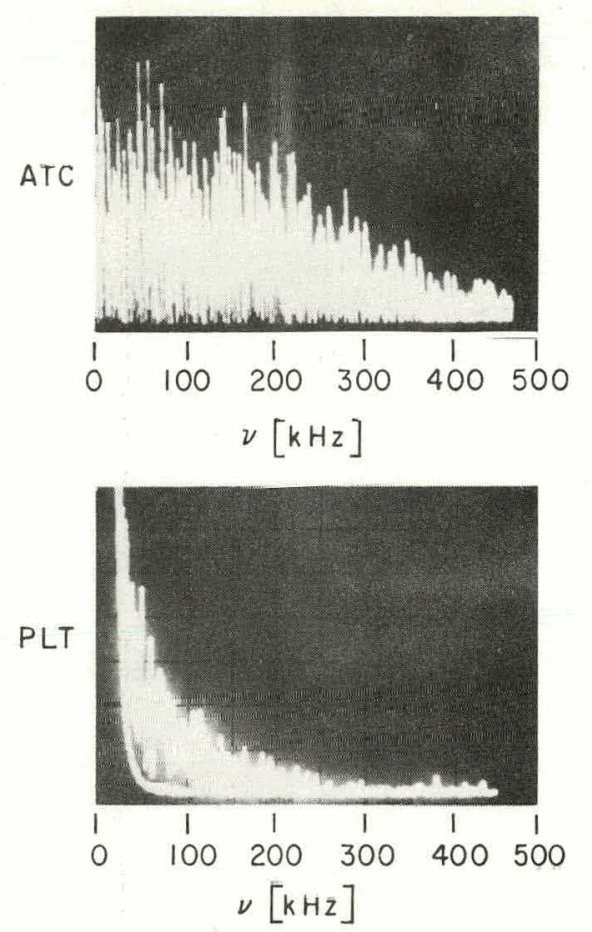

773649

Fig. 6. Frequency width at half intensity of $\mathrm{S}(\mathrm{k}, \omega)$ : - ATC; 0 PLT (with peake $\bar{d} \mathrm{~T}_{\mathrm{e}}$ profile).
773646

Fig. 5. Frequency spectrum of fluctuations with $\mathrm{k} \simeq 10 \mathrm{~cm}^{-1}$ in ATC (top) and PLT (bottom).

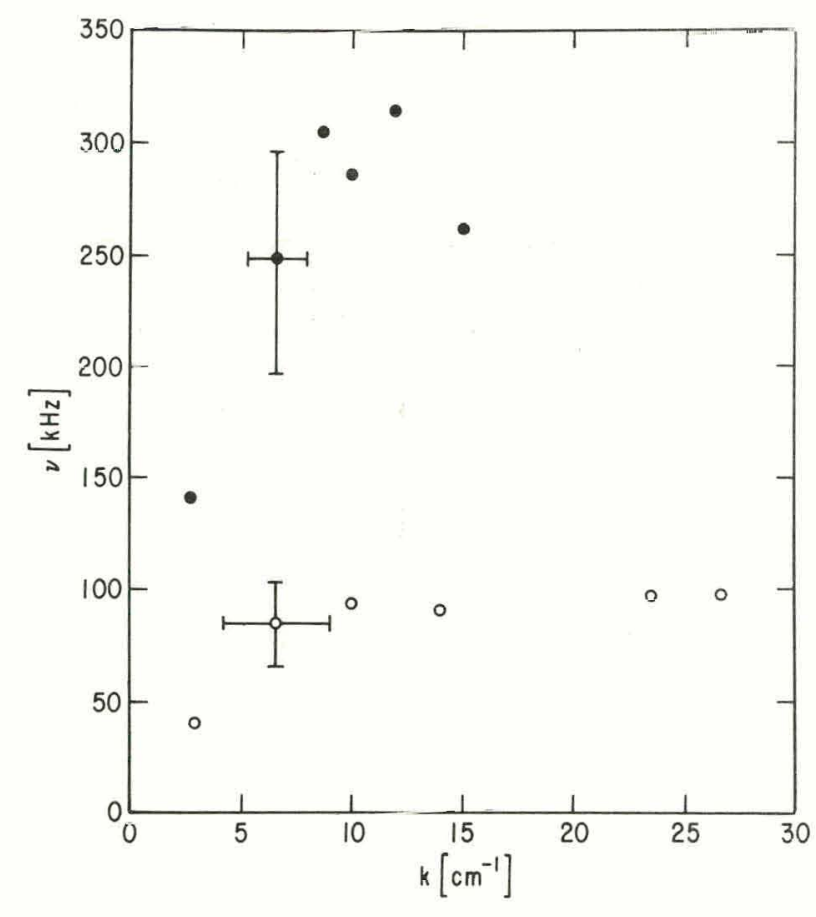


773650

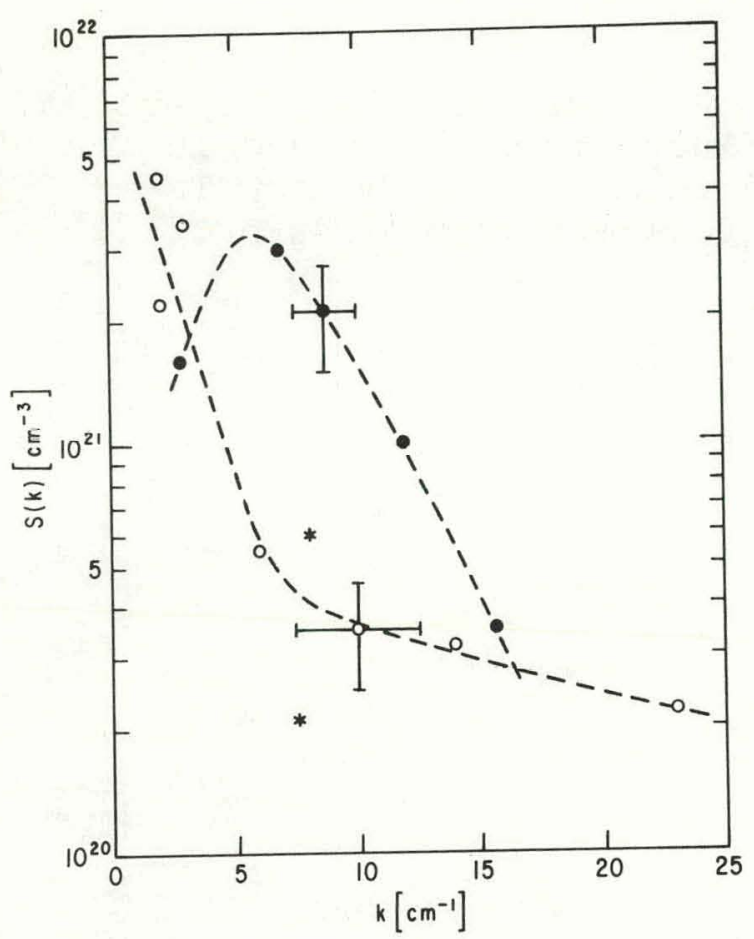

Fig. 7. Spectral density of fluctuations: 0 - PLT (at $\left.\theta=90^{\circ}\right)$; * PLT (at $\theta=-54^{\circ}$ and $\theta=-135^{\circ}$ ); ATC.

773648

Fig. 8. Spectral density of flucLuations in PIT with hollow $\mathrm{T}_{e}$-profile: $0-\theta=90^{\circ} ; *-\theta=-54^{\circ}$ (larger amplitude) and $\theta=-135^{\circ}$ (smaller amplitude).

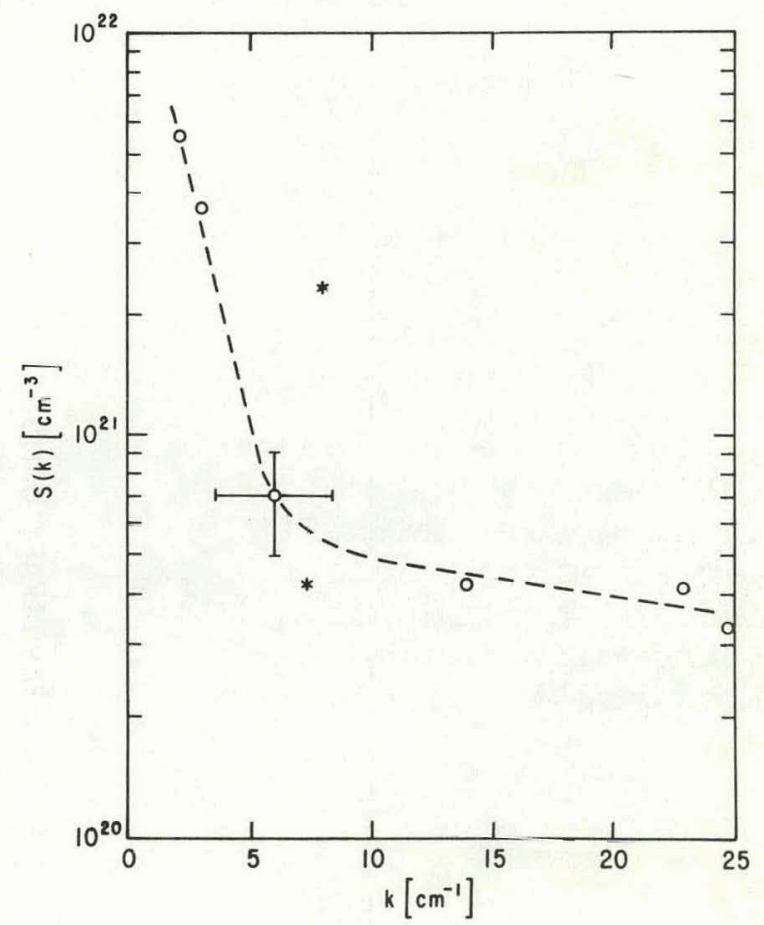




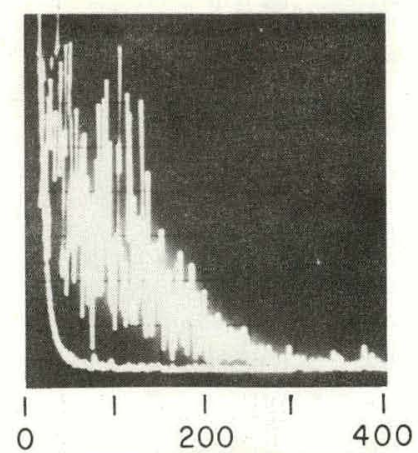

773647

Fig. 9. Frequency spectrum of fluctuations observed in the PLT discharge with hollow $\mathrm{T}_{\mathrm{e}}$-profile (top) and peaked $\mathrm{T}_{\mathrm{e}}$-profile (bottom)

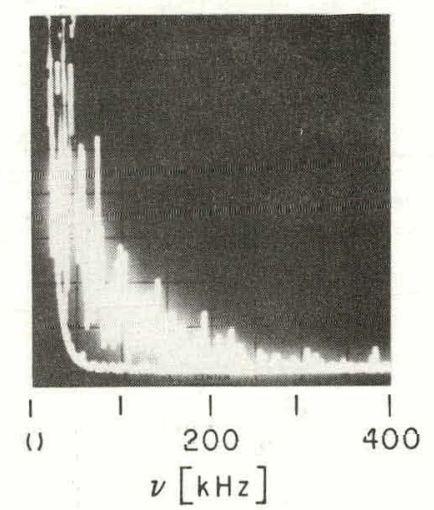

773654

Fig. 10. Time evolution of $\mathrm{S}(\mathrm{k}) \quad\left(\right.$ with $\mathrm{k} \simeq 8 \mathrm{~cm}^{-1}$ ) which was observed in PLT discharges with a hollow $\mathrm{T}_{\mathrm{e}}$-profile (continuous line) and with peaked $\mathrm{T}_{\mathrm{e}}$ profile (broken line).

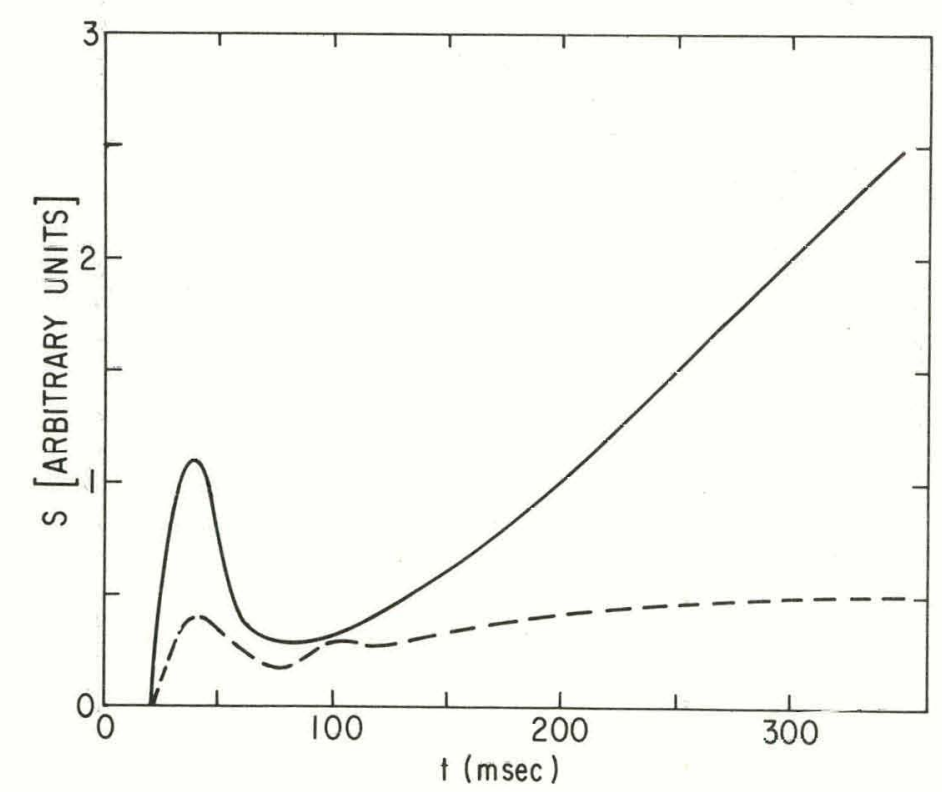




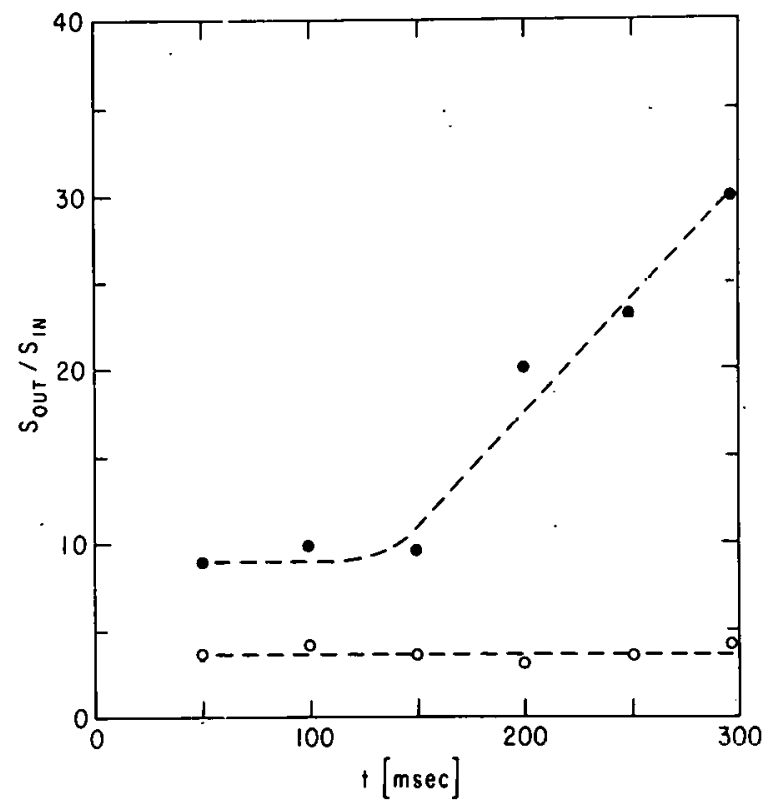

773653

Fig. 11. Ratio of the outside value of $S(k, \omega)$ to the inside one $\left[(\omega / 2 \pi)=1 \overline{0} 0 \mathrm{kHz}\right.$ and $\left.\mathrm{k} \simeq 8 \mathrm{~cm}^{-1}\right]$ which was observed in PLT discharges with a hollow $\mathrm{T}_{\mathrm{e}}$-profile (o-points) and with a peaked $\mathrm{T}_{e^{-}}$ profile (o-points). 